\title{
Inflammation Spreading from Subacute Thyroiditis Following Fine-needle Aspiration: A Case Report
}

\author{
Bowen Zheng, MD ${ }^{a}$, Tao $W u, M D^{a}$, Ying Ruan, MD ${ }^{b}$, Jun Chen, MD ${ }^{a}$, Jie Ren, MD ${ }^{a, *}$ \\ ${ }^{a}$ Department of Medical Ultrasonics, Guangdong Province Key Laboratory of Hepatology Research, the Third Affiliated Hospital of Sun \\ Yat-Sen University, Guangzhou, 510630, China; ${ }^{b}$ Department of Thyroid and Breast Surgery, Guangdong Province Key Laboratory of \\ Hepatology Research, the Third Affiliated Hospital of Sun Yat-Sen University, Guangzhou, 510630, China \\ Received March 27, 2019; revision requested May 27, 2019; revision received June 1; accepted June 4.
}

\begin{abstract}
Ultrasound guided fine-needle aspiration (FNA) is well-accepted in the evaluation of thyroid nodules. Though local pain and minor hematomas are the most common complications, other adverse events following FNA are rare. In this case report, we described a complication showing inflammation spreading in a healthy woman after FNA. We presented the case to make physicians aware of the potential complication.
\end{abstract}

Key Words: Thyroid; Biopsy, Fine-Needle; Thyroiditis, Subacute; Complication; Inflammation

Advanced Ultrasound in Diagnosis and Therapy 2019;02:050-052

DOI: $10.37015 / A U D T .2019 .190810$

$\mathrm{U}$ ltrasound guided fine-needle aspiration (FNA) is primarily recommended for evaluation of thyroid nodules and has been well-accepted due to its simplicity, reliability and safety [1]. Local pain and minor hematomas are the most common complications [1-3]; while serious complications seem to be extremely rare. Other reported adverse events following thyroid FNA include swelling of thyroid, infection, recurrent laryngeal nerve palsy, vasovagal reaction, tracheal puncture, dysphagia, needle track seeding, needle track sinus, nodule volume alteration, and post-aspiration thyrotoxicosis [1]. Here we encountered a case of complication following FNA, showing inflammation spreading in the thyroid in a healthy woman. We presented the case to make physicians aware of the potential complication.

\section{Case Presentation}

A 37-year-old woman was referred for an incidental thyroid nodule in the right lobe. No fever, upper airway infection or local pain was noted. Her medical history was not remarkable for any radiation exposure to the cervical area or for a family history of thyroid disease.
Laboratory examinations revealed slightly elevated thyroglobulin $(\mathrm{TG})$ antibody $(5.07 \mathrm{IU} / \mathrm{ml}, \mathrm{N}:<4.11)$ and decreased thyroid stimulating hormone $(0.062 \mathrm{uIU} /$ $\mathrm{ml}, \mathrm{N}: 0.35-4.94$ ), while the results of thyroxin, thyroid peroxidase antibody, erythrocyte sedimentation rate and complete blood count were within normal ranges. The US showed a heterogeneous hypoechoic nodule of $17 \times 8 \times 24 \mathrm{~mm}$ in size in the right lobe of thyroid, with indistinct margin. Color Doppler showed lack of color flow in the nodule (Fig. A and B). The clinical diagnosis was thyroiditis $[4,5]$, which was scored as TI-RADS category 4 [6], without regional lymphadenopathy. USguided FNA, using a $25 \mathrm{G}$ needle by lateral approach was performed to rule out malignancy. The skin was cleaned with compound iodine. The needle was inserted through the surrounding thyroid into the targeted nodule. Five passes were performed systematically to ensure adequate specimen for cytological analysis. The result was reported as Bethesda II (benign), which showed benign follicular cells in the background of erythrocyte and inflammatory cells (Fig. C). Thyroiditis was considered as a possible diagnosis and US follow-up was advised.

\footnotetext{
* Corresponding author: Department of Medical Ultrasonics, Guangdong Province Key Laboratory of Hepatology Research, the Third Affiliated Hospital of Sun Yat-Sen University, Guangzhou, 510630, China

e-mail: renjieguangzhou@126.com
} 


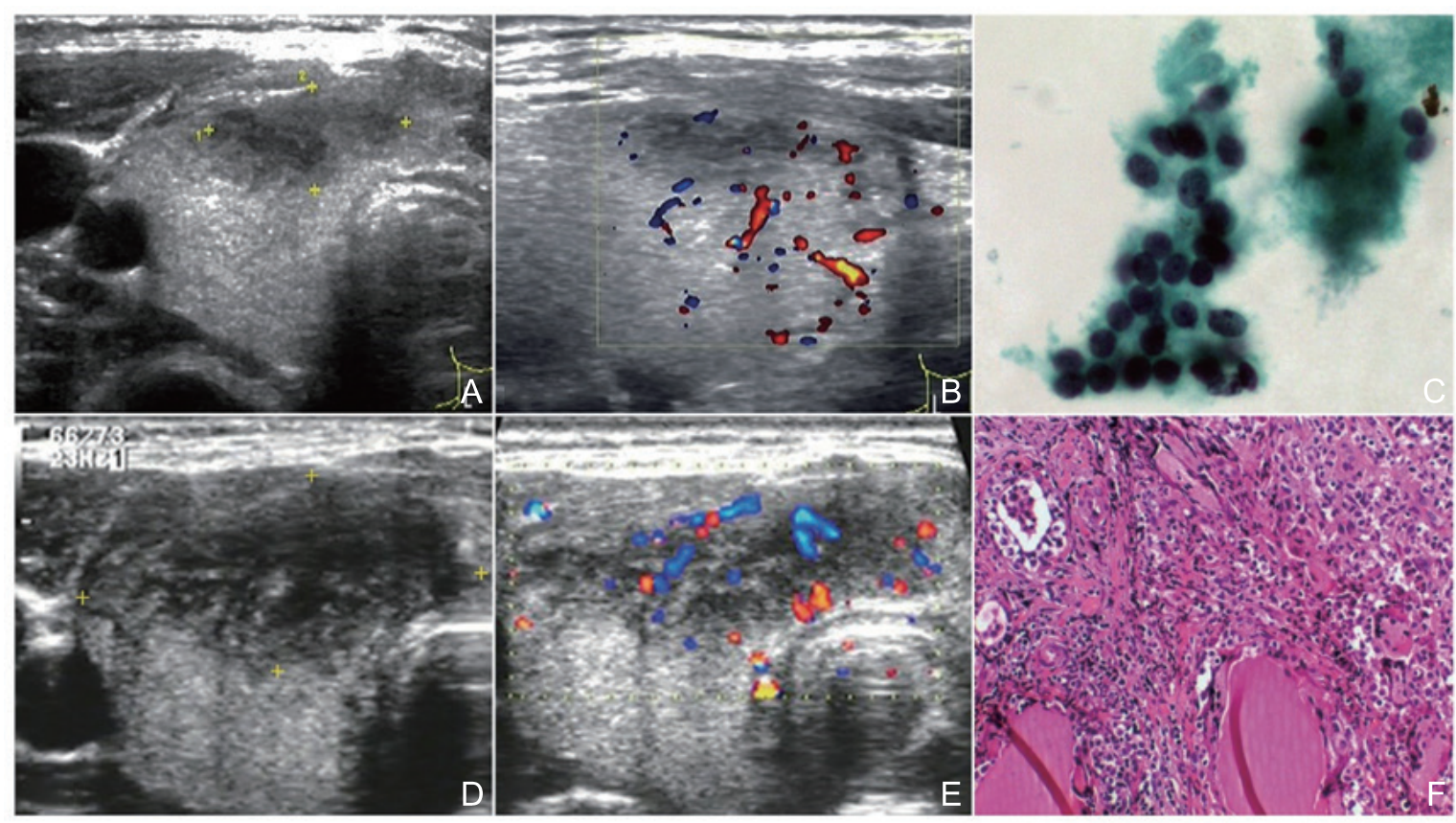

Figure 1 US showed a $17 \times 8 \times 24 \mathrm{~mm}$ nodule in the right lobe of thyroid showing a heterogeneous, hypoechoic area with indistinct margin (A, +) and lack of color flow (B) before fine-needle aspiration (FNA). Cytological analysis reported Bethesda II (benign), showing benign follicular cells (C). US showed obviously enlargement of the right-sided nodule $(34 \times 13 \times 33 \mathrm{~mm})$ spreading to the isthmus $(\mathrm{D},+)$, with increased of color flow (E) 12 days after FNA. Histological examination revealed subacute thyroiditis $(\mathrm{F})$.

12 days after the FNA, the patient was admitted to hospital with complaints of pain, swelling and palpable firm mass in the right neck. Physical examination indicated a hard and swollen mass in the right lobe of thyroid without erythema or skin temperature arisen. Body temperature and complete blood count were within normal ranges, while the neutrophil percentage increased $(78.9 \%, \mathrm{~N}: 40.0 \%-75.0 \%)$ and lymphocyte percentage decreased $(14.7 \%, \mathrm{~N}: 20.0 \%-50.0 \%)$. US examination of the thyroid showed obvious enlargement of the right-sided nodule $(34 \times 13 \times 33 \mathrm{~mm})$, which spread to the isthmus, with increasing color flow (Fig. D-E). Though inflammation spreading related to FNA was suspected first, the patient was anxious for malignancy and required surgery. The partial thyroidectomy was then performed and the histological examination revealed subacute thyroiditis (SAT) in the right lobe, which showed disrupted follicles infiltrated by inflammatory cells including multinucleated giant cells (MNGCs), lymphocytes, and neutrophils (Fig. F).

\section{Discussion}

This is a rare case of complication, showing inflammation spreading in SAT after FNA in a healthy individual.

SAT is an inflammatory condition of the thyroid with characteristic presentations and clinical course, which includes classic painful SAT (PFSAT), and painless SAT (PLSAT) [7]. Unlike PFSAT showing a tendency to follow upper respiratory tract infections or sore throats, which has suggested a viral infection, PLSAT occurs spontaneously or following pregnancy. It is histologically similar to Hashimoto's thyroiditis and shows thyroid enlargement usually without discomfort, positive anti-thyroid antibodies, and thyroid function test abnormalities. Though PLSAT is less common than PFSAT, it should be considered in the differential diagnosis of a solitary, painless nodule [8].

However, PLSAT appearing as painless solid nodules indeed generates challenges in differentiating from malignancy [9]. FNA may provide assistance, particularly in excluding other thyroid lesions [10], as it shows characteristic for SAT, including the presence of multiple MNGCs, a dirty background accompanied by mild-moderate cellularity, degenerated-proliferated follicular epithelium cells, rare epithelioid granulomas and mixed type inflammatory cells $[9,11]$. Although SAT is usually diagnosed clinically, the assessment of US and cytological findings in conjunction with clinical findings will assist in the achievement of an accurate diagnosis [9].

The inflammation spreading in SAT following FNA hasn't been reported and the reasons of the inflammation spreading remain unclear. One possible explanation is that an autoimmune reaction, which has already existed in PLSAT as these patients are frequently thyroid peroxidase (TPO) or TG antibody 
positive [7], may be intensified by FNA which is a kind of mechanical stimulation, to cause more immune cells to infiltrate and spread [12] in this present case. FNArelated infection may be another explanation. Unlike those reported cases of post-FNA infection showing acute suppurative thyroiditis or thyroid abscess [1315], there wasn't sufficient evidence, such as high fever, leukocytosis, or abscess formation, to support common bacterial secondary infection following FNA, like Propionibacterium acnes [14] in the present case. However, infection caused by uncommon bacteria might exist, since the patient showed increased neutrophil percentage and decreased lymphocyte percentage after FNA.

This study describes a case of inflammation spreading developed in a healthy individual with PLSAT after FNA. The possibility of such an extremely rare complication should be kept in mind.

\section{Conflicts of Interest}

The authors declare that they have no competing interests.

\section{References}

[1] Polyzos SA, Anastasilakis AD. Clinical complications following thyroid fine-needle biopsy: a systematic review. Clin Endocrinol (Oxf) 2009;71:157-65.

[2] Gharib H, Papini E, Valcavi R, Baskin HJ, Crescenzi A, Dottorini ME, et al. American Association of Clinical Endocrinologists and Associazione Medici Endocrinologi medical guidelines for clinical practice for the diagnosis and management of thyroid nodules. Endocr Pract 2006;12:63-102.

[3] Wu M, Burstein DE. Fine needle aspiration. Cancer Invest 2004;22:620-628.

[4] Cappelli C, Pirola I, Gandossi E, Formenti AM, Agosti B, Castellano M. Ultrasound findings of subacute thyroiditis: a single institution retrospective review. Acta Radiol 2014; 55:429-433.

[5] Park SY, Kim EK, Kim MJ, Kim BM, Oh KK, Hong SW, et al. Ultrasonographic characteristics of subacute granulomatous thyroiditis. Korean J Radiol 2006;7:229-234.

[6] Tessler FN, Middleton WD, Grant EG, Hoang JK, Berland LL, Teefey SA, et al. ACR Thyroid Imaging, Reporting and Data System (TI-RADS): White Paper of the ACR TI-RADS Committee. J Am Coll Radiol 2017;14:587-595.

[7] Hennessey J. Subacute Thyroiditis. In: De Groot LJ, Chrousos G, Dungan K, et al., eds. Endotext. South Dartmouth (MA): MDText. com, Inc.; 2000.

[8] Bianda T, Schmid C. De Quervain's subacute thyroiditis presenting as a painless solitary thyroid nodule. Postgrad Med J 1998;74:602603.

[9] Vural C, Paksoy N, Gok ND, Yazal K. Subacute granulomatous (De Quervain's) thyroiditis: Fine-needle aspiration cytology and ultrasonographic characteristics of 21 cases. Cytojournal 2015;12: 9.

[10] Garcia Solano J, Gimenez Bascunana A, Sola Perez J, Campos Fernandez J, Martinez Parra D, Sanchez Sanchez C, et al. Fine-needle aspiration of subacute granulomatous thyroiditis (De Quervain's thyroiditis): a clinico-cytologic review of 36 cases. Diagn Cytopathol 1997; 16:214-220.

[11] Lamichaney R, Sherpa M, Das D, Bhutia CT, Laishram S. FineNeedle Aspiration of De Quervain's Thyroiditis (Subacute Granulomatous Thyroiditis): A Cytological Review of 20 Cases. $J$ Clin Diagn Res 2017;11:Ec09-Ec11.

[12] McWhorter FY, Davis CT, Liu WF. Physical and mechanical regulation of macrophage phenotype and function. Cell Mol Life Sci 2015;72:1303-1316.

[13] Unluturk U, Ceyhan K, Corapcioglu D. Acute suppurative thyroiditis following fine-needle aspiration biopsy in an immunocompetent patient. J Clin Ultrasound 2014;42:215-218.

[14] Sun JH, Chang HY, Chen KW, Lin KD, Lin JD, Hsueh C. Anaerobic thyroid abscess from a thyroid cyst after fine-needle aspiration. Head Neck 2002;24:84-86.

[15] Chen HW, Tseng FY, Su DH, Chang YL, Chang TC. Secondary infection and ischemic necrosis after fine needle aspiration for a painful papillary thyroid carcinoma: a case report. Acta Cytol 2006;50:217-220. 